\title{
Self-Healing during Electrical Treeing: A Feature of the Two-Phase Liquid-Solid Nature of Silicone Gels
}

\author{
L. M. Salvatierra(a,b), L. I. Kovalevski(a,b), P. L. Dammig Quiña ${ }^{(a)}$, I. M. Irurzun ${ }^{(a)}$, E. E. Mola ${ }^{(a, b)}$ \\ (a) CCT La Plata CONICET. Instituto de Investigaciones Fisicoquímicas Teóricas y Aplicadas (INIFTA), Facultad de Ciencias \\ Exactas, Universidad Nacional de La Plata. \\ Diagonal 113 y 64, CP (1900), La Plata, Argentina. \\ (b) Facultad de Química e Ingeniería, Pontificia Universidad Católica Argentina. \\ Av. Pellegrini 3314, CP (2000), Rosario, Argentina.
}

\author{
and S. J. Dodd, L. A. Dissado \\ University of Leicester, \\ Department of Engineering, \\ Leicester LE1 7RH, UK.
}

\begin{abstract}
We report the results of electrical treeing tests carried out on a range of silicone dielectric gel samples made by varying the ratio of the same two components used for cross-linking a commercial silicone gel. These samples range from liquids to elastomers via two-phase gels. The extent of the curing is followed by means of FTIR spectroscopy and the samples are characterized through their dynamical mechanical properties. It is shown that the gel samples exhibit treeing behavior that includes features typical of both liquids and solids. A filamentary structure is produced that has permanence together with attached bubbles that self-heal locally during treeing, although differences in detail were found depending on the degree of curing. Removal of the treeing voltage allows a more substantial self-healing to occur. This behavior is discussed in terms of the two-phase liquid-solid nature of the silicone gels.
\end{abstract}

\section{Index Terms — Silicone Polymer Gels, Trees, Partial Discharges.}

\section{INTRODUCTION}

SILICONE materials are widely employed in an enormous variety of industrial applications because they have excellent dielectric properties, chemical stability, and great versatility. Their versatility arises because their molecular microstructure can be easily manipulated from liquids (as used in some HV power transformer cooling systems), to solids (elastomeric housing, insulators, cables, panels, surface treatments, joints, etc.), leading to an enormous variety of physicochemical properties. Silicone gels are one such variation. Although the applications for silicone gels in industry are not the most widely occurring they are interesting dielectric materials because they cure in place to form cushioning, self-healing and resilient materials. They are therefore used as a special class of encapsulants of power electronics (i.e. insulated-gate bipolar transistors or IGBTs), protecting them from mechanical and thermal shocks, moisture and other contaminants. The special feature of a gel is that when cured it retains much of the stress relief and self-healing qualities of a liquid while providing the dimensional stability of a cross-linked elastomer. Their physical and electrical stability is maintained over a wider temperature range $\left(-45^{\circ} \mathrm{C}\right.$ to $\left.200^{\circ} \mathrm{C}\right)$ than other polymers [1-3].
The self-healing capability of silicone gels is of some importance in high voltage (HV) applications where partial selfhealing has been observed when the gel was subjected to partial discharges (PDs) [2-4]. In a solid polymer, a cavity resulting from PDs will be a permanent defect and usually will propagate and grow as a fractal or tree structure leading eventually to failure. In liquids, PDs cause jets or "streamer" projections from an initiating point electrode constituted by ionized gaseous cavities that disappear quickly, usually after some microseconds $(\mu \mathrm{s})$ [5] at atmospheric pressure. The semi-liquid, semi-solid, structure of a silicone gel means that both of these features can be expected when it is exposed to fields high enough to cause electrical tree formation [4]. For this reason silicone gels are interesting to study in terms of the formation of electrical trees and sample failure, and also electrical ageing.

Some dielectric silicone gels available in the market are a 1:1 mixture of two liquid components (parts A and B). The mixture is cured to ensure the final desired sticky and soft polymer, with a curing time that depends on the curing temperature. In a preliminary study [4], we presented results for these gels showing the self-healing behavior that is observed under electrical treeing testing. In this work we purposely change this 
ratio, obtaining a continuous set of samples with different physicochemical properties. Samples with ratios between 1:0 (pure liquid of part A) and 0:1 (pure liquid of part B) have been studied using FTIR and dynamical mechanical analysis to characterize the physicochemical nature of the samples. FTIR shows that the maximum amount of cross-linking was not located at the 1:1 ratio used for the gels previously studied [4]. Unexpectedly therefore increasing part A close to 2:1 ratio yielded a highly cross-linked silicone elastomer, reaching the highest curing efficiency between active agents inside each liquid precursor component. Dynamic mechanical analysis allowed us to identify the composition ranges that gave elastomers, gels, or liquids. This very rich set of samples, obtained with the same polymer product, allows us to attempt a deeper interpretation of the self-healing phenomena in polymers when they are subjected to electrical treeing tests.

\section{EXPERIMENTAL PROCEDURES}

\subsection{SAMPLE MATERIAL}

A commercial dielectric silicone gel from RS Components Ltd. UK, is obtained as the result of a cross-linking reaction between two transparent liquid components (termed part A and part B). According to the manufacturer's data sheet, they should be mixed in a 1 to 1 ratio to ensure that a gel system is reached. However, we found that we could also obtain different microstructures (cross-linking densities) by modifying the A to $\mathrm{B}$ ratio. We therefore prepared a range of samples by varying the ratios from 1:0 (pure liquid of part $A$ ) to $0: 1$ (pure liquid of part $\mathrm{B}$ ), passing through the 1:1 gel condition as offered by the supplier.

\subsection{SAMPLE PREPARATION}

During preparation a given quantity of the components were manually mixed for 3 minutes. For electrical treeing tests, the liquid components were degasified $2 \mathrm{~min}$ in vacuum $(10-100 \mathrm{mmHg})$ at room temperature $\left(\mathrm{RT}=22^{\circ} \mathrm{C}\right)$. Large bubbles formed almost immediately and these were collapsed by introducing atmospheric pressure and then continuing the vacuum. The mixed liquids were then cast into transparent polystyrene cuvettes of $10 \mathrm{~mm} \times 10 \mathrm{~mm}$ x $40 \mathrm{~mm}$ of which the base had previously been removed and replaced by a plane film metal electrode (aluminum foil). Ogura Jewels Ltd. steel needles of $5 \mu \mathrm{m}$ tip radius were embedded in each fully filled liquid cuvette and positioned with a fixed gap of $3 \mathrm{~mm}$ between the pin tip and film metal electrode. The specimens were cured for 4 hours at $65^{\circ} \mathrm{C}$ in an inert atmosphere. Table 1 shows the sample nomenclature as a percentage of component A.

\subsection{SAMPLE TESTING}

\subsubsection{Infrared Absorption (FTIR)}

Samples were analyzed in a Perkin Elmer Spectrum One FTIR spectrometer. The two components part A and part B were measured separately by deposition onto a $\mathrm{NaCl}$ cell. In addition, in-situ curing for different samples of Table 1 was measured at times of $0,2,4,10$ and 60 minutes following 3 min of mixing and 2 min of degasification.

Table 1. Sample's nomenclature and testing (FTIR: Infrared absorption tested; M: Mechanical tested; ET: Electrical Treeing tested)

\begin{tabular}{|c|c|c|c|c|}
\hline $\begin{array}{c}\text { Sample } \\
(\% \text { part A) }\end{array}$ & Ratio A:B & FTIR & M & ET \\
\hline $0 \%$ & $0: 1$ & $\bullet$ & & $\bullet$ \\
\hline $25 \%$ & $1: 3$ & & & $\bullet$ \\
\hline $33 \%$ & $1: 2$ & & & $\bullet$ \\
\hline $40 \%$ & $1: 1.5$ & & & $\bullet$ \\
\hline $45 \%$ & $1: 1.22$ & & $\bullet$ & $\bullet$ \\
\hline $50 \%$ & $1: 1\left(^{*}\right)$ & $\bullet$ & $\bullet$ & $\bullet$ \\
\hline $55 \%$ & $1.22: 1$ & & $\bullet$ & $\bullet$ \\
\hline $60 \%$ & $1.5: 1$ & & $\bullet$ & $\bullet$ \\
\hline $66 \%$ & $2: 1$ & & $\bullet$ & $\bullet$ \\
\hline $70 \%$ & $2.33: 1$ & $\bullet$ & $\bullet$ & $\bullet$ \\
\hline $75 \%$ & $3: 1$ & & $\bullet$ & $\bullet$ \\
\hline $80 \%$ & $4: 1$ & $\bullet$ & $\bullet$ & $\bullet$ \\
\hline $90 \%$ & $9: 1$ & $\bullet$ & $\bullet$ & $\bullet$ \\
\hline $100 \%$ & $1: 0$ & $\bullet$ & & $\bullet$ \\
\hline
\end{tabular}

(*) ratio suggested by the supplier to obtain a dielectric silicone gel product as it is offered in the market.

\subsubsection{Mechanical analysis}

Rheological studies were performed with an AR-G2 Rheometer from TA Instruments. The samples defined in Table 1 were placed and cured in situ in the rheometer following also $3 \mathrm{~min}$ of mixing and $2 \mathrm{~min}$ of degasification. They were measured isothermally at $65^{\circ} \mathrm{C}$ during curing with a maximum shear strain of $1 \%$, at $0.2 \mathrm{~Hz}$. After completion of the cure, frequency scans at different temperatures were carried out. Both parts of complex shear modulus (G' and G' ') and the loss tangent $\left(\tan (\delta)=G^{\prime} ' / G^{\prime}\right)$, were recorded for further analysis.

\subsubsection{Electrical treeing}

A typical experimental array for electrical treeing was used. The cuvette with the pin-plane sample was placed inside a faraday cage. The voltage applied to the pin could be varied using a manual variac controlling an internal $20 \mathrm{kV}$ transformer at $50 \mathrm{~Hz}$. The plane electrode was connected to earth. A light source placed behind the sample allowed back illuminated images of the electrical trees to be recorded using a CMOS full-frame high definition (HD: 1920px x 1080px) DSLR camera. The camera allowed video recording at $20 \mathrm{fps}$ in HD.

\section{RESULTS}

\subsection{INFRARED ABSORPTION (FTIR)}

For dielectric applications an addition curing reaction is used to achieve cross-linking, by using vinyl end-blocked molecular chains in one component, which react with the other component containing $\equiv \mathrm{SiH}$ groups carried by functional oligomers $[1,6]$. The addition occurs mainly on the terminal carbon and is catalysed by platinum metal complexes, preferably organometallic compounds to enhance their compatibility. It has been suggested that the mechanism is an oxidative addition of the $\equiv \mathrm{SiH}$ onto the platinum, followed by hydrogen atom transfer on the double bond, and 
reductive elimination of the product $[1,6]$. There is no byproduct with this reaction, and moulded pieces made with a product using this cure mechanism retain their integrity very well (no shrinkage, and no presence of condensation products).

In Figure 1a, it can be seen that both components $\mathrm{A}$ and $\mathrm{B}$ exhibit a similar spectrum. In fact, they seem to be basically the same silicone oil-liquid, excepting in the groups active in curing. Component A shows a medium intensity absorption peak at $2127 \mathrm{~cm}^{-1}$ related to the $\equiv \mathrm{SiH}$ oligomer, which is absent in component B though there seems to be a spurious absorbance of very weak intensity at the same wavenumber, which should not be the same group. Component B has the platinum catalyst and the vinyl end-blocked molecular chains, but these are not observed through the FTIR, possibly due to an overlapping effect. The Si-O-Si backbone produces the broad band with two maxima absorbance at 1092 and 1018 $\mathrm{cm}^{-1}$. The methyl deformation at $1261 \mathrm{~cm}^{-1}$ and the asymmetric stretch at $2964 \mathrm{~cm}^{-1}$ are also strong and sharp. The methyl rocking motion with contribution from the $\mathrm{Si}-\mathrm{C}$ stretch at $798 \mathrm{~cm}^{-1}$ is also strong.
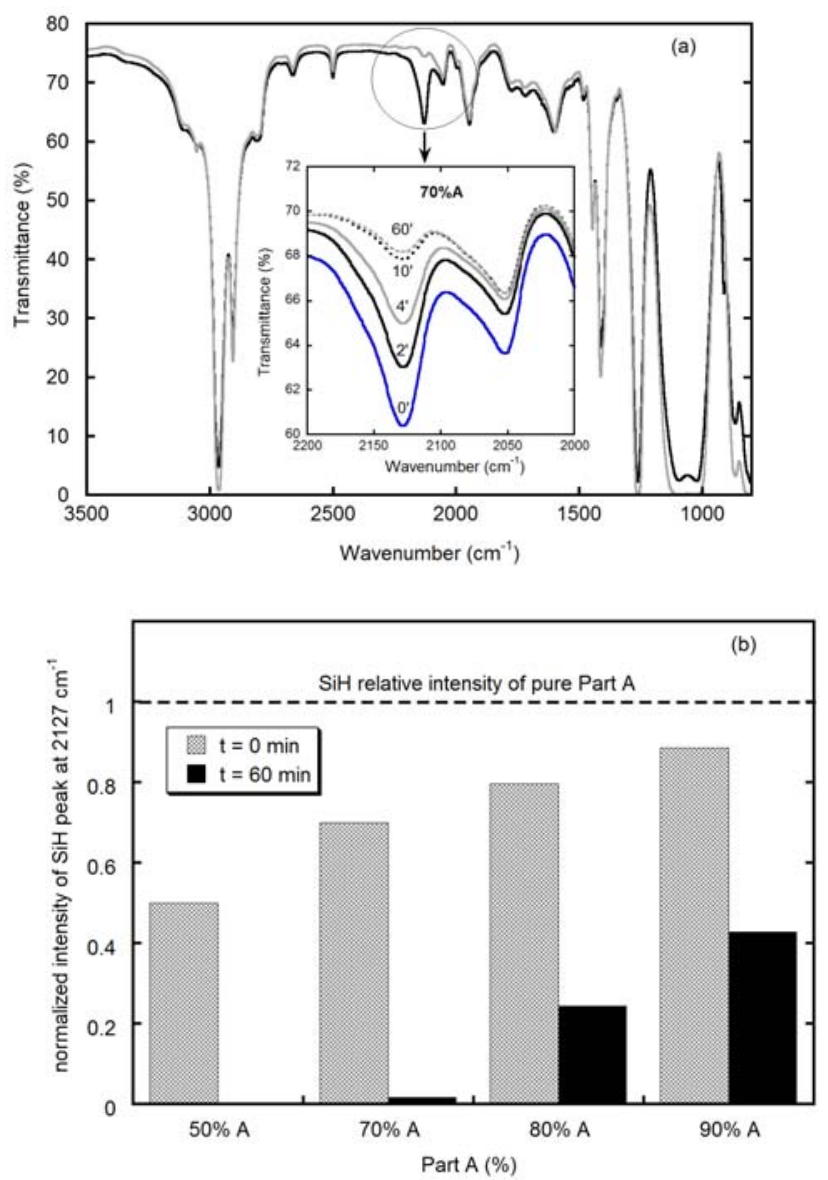

Figure 1. (a) FTIR spectra of part A (black) and B (grey). The inset shows the consumption of the $\equiv \mathrm{SiH}$ peak at $2127 \mathrm{~cm}^{-1}$ for the $70 \% \mathrm{~A}$ sample, at different times (in minutes) after 5 min of mixing the components and degasification. (b) Normalized intensity of $\equiv \mathrm{SiH}$ radical at the $2127 \mathrm{~cm}^{-1}$ peak relative to the $1945 \mathrm{~cm}^{-1}$ band for a range of compositions measured at $\mathrm{t}=0$ $\min$ and $t=60 \mathrm{~min}$, after a preparation time of $5 \mathrm{~min}$. The value for pure component $\mathrm{A}$ is shown as the maximum possible.
The inset to Figure 1a shows the disappearance of the $\equiv \mathrm{SiH}$ absorption band at $2127 \mathrm{~cm}^{-1}$ of the $70 \% \mathrm{~A}$ sample during curing (i.e. after $3 \mathrm{~min}$ of manual mixing and $2 \mathrm{~min}$ of degasification).

Figure $1 \mathrm{~b}$ shows the radical $(\equiv \mathrm{SiH})$ consumption process during the curing of a range of compositions $(50 \% \mathrm{~A}$ to $90 \% \mathrm{~A}$ ) by means of the ratio of the intensity of the $2127 \mathrm{~cm}^{-1}$ absorption peak to that of the $1945 \mathrm{~cm}^{-1}$ band. The value for pure component $\mathrm{A}$ is also shown as defining the maximum possible value of this measure. As can be observed all the relative amounts of $\equiv \mathrm{SiH}$ calculated from the FTIR at $\mathrm{t}=0$ min match with the respective percentage of component $\mathrm{A}$ during mixing (i.e. this relative absorption intensity is a good quality-check of the initial ratio). Figure $1 \mathrm{~b}$ also shows that $\equiv \mathrm{SiH}$ is consumed (i.e. reacted to form cross-links) during the curing process with the radical being fully consumed after 60 minutes in the case of the $50 \% \mathrm{~A}$ sample. In samples with bigger concentrations of component A not all of the $\equiv \mathrm{SiH}$ has reacted by the end of the curing process at 60 minutes, i.e. $70 \% \mathrm{~A}$ has only a little unreacted amount at this time, and the unreacted quantity increases as the percentage of A increases. Moreover, for samples with more than $70 \% \mathrm{~A}$ the amount of unreacted $\equiv \mathrm{SiH}$ increases at twice the rate that the percentage of $\mathrm{A}$ increases, i.e. an increase of $10 \%$ in A gives an increase of unreacted $\equiv \mathrm{SiH}$ radical at the end of the reaction of $20 \%$. The amount of unreacted $\equiv \mathrm{SiH}$ can be related to the fraction of component $\mathrm{A}$ in the mixture (for samples with $70 \% \mathrm{~A}$ or more) by the following linear expression using the method of least squares to fit the data.

$$
y=2.059 x-1.418
$$

Here $y$ is the normalized relative intensity of $\equiv \mathrm{SiH}$ radical and $x$ is the fraction of component $\mathrm{A}$. This expression implies that the $\equiv \mathrm{SiH}$ radical is fully consumed for mixtures with concentrations of A up to $68.9 \%$, since $y=0$ when $x=0.689$. Thus this is the largest fraction of $\mathrm{A}$ for which all $\equiv \mathrm{SiH}$ radicals are consumed in cross-links, which defines the highest efficiency ratio for the cross-linking reaction.

\subsection{MECHANICAL ANALYSIS}

Figure 2 shows the evolution of the real component of the elastic shear modulus $\left(\mathrm{G}^{\prime}\right)$ at a frequency of $0.2 \mathrm{~Hz}$ during the curing process at $65^{\circ} \mathrm{C}$ for samples between $45 \% \mathrm{~A}$ and $90 \% \mathrm{~A}$ (see Table 1). As can be observed the final cured state increases in hardness between $45 \% \mathrm{~A}$ and $66-70 \% \mathrm{~A}$ and then reduces steadily as the percentage A continues to increase up to $90 \%$. Another distinctive characteristic is that the initial slope $\mathrm{dG} / \mathrm{dt}$ during the curing reaction increases steadily until the highest modulus is reached in samples $66 \% \mathrm{~A}$ and $70 \% \mathrm{~A}$. Although the final modulus reduces thereafter the initial slope remains higher than a sample with smaller percentage of $\mathrm{A}$ and similar G' end-value: e.g. compare sample $55 \% \mathrm{~A}$ with $90 \% \mathrm{~A}$, and sample $60 \% \mathrm{~A}$ with $80 \% \mathrm{~A}$. 


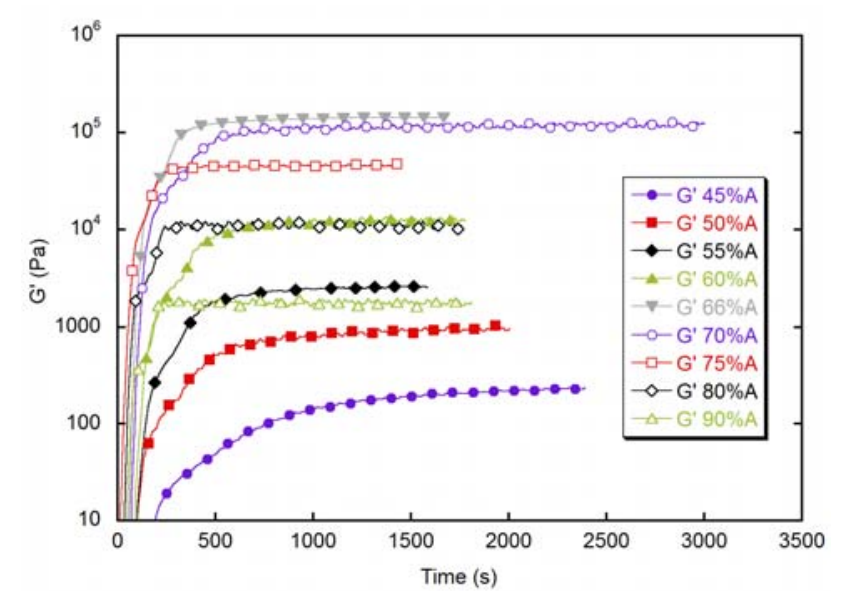

Figure 2. Evolution of $\mathrm{G}^{\prime}$ during curing at $65^{\circ} \mathrm{C}, 0.2 \mathrm{~Hz}$ and a maximum shear strain of $1 \%$, for samples $45 \%, 50 \%, 55 \%, 60 \%, 66 \%, 70 \%, 75 \%, 80 \%$ and $90 \%$ of component $\mathrm{A}$.

Figure 3 shows the final stabilized end-values of $\mathrm{G}^{\prime}$ (Figure 2) and $\tan (\delta)$ after curing at $65^{\circ} \mathrm{C}$ as a function of concentration of $\mathrm{A}$. The shaded regions at $<40 \% \mathrm{~A}$ and $>95 \% \mathrm{~A}$ correspond to a liquid phase, where the mixed components cannot reach a gel point. In these cases the value of $\mathrm{G}^{\prime}$ ' is always higher than that of G', giving a $\tan (\delta)>1$ (i.e. the sample behaves more as a high viscous liquid material than an elastic solid). Therefore, the only cured samples included in the figure are those that lie between these liquid regions. It is easy to see that there is a maximum in the hardening at $66-70 \% \mathrm{~A}$ that corresponds to the maximum efficiency of crosslink generation between components A and $\mathrm{B}$, and which produces a rubbery-like elastomer. The magnitude of $G^{\prime}$ at this composition is more than two orders of magnitude larger than its value for the silicone gel-like product at $50 \% \mathrm{~A}(1: 1)$ specified by the supplier in the datasheet. The further decrease in the $G^{\prime}$ value for compositions containing more than $70 \% \mathrm{~A}$ is attributed to the reduced amount of cross-linking reactions that can be achieved, when an excess of $\mathrm{A}$ is added, as revealed by the residual amount of $\equiv \mathrm{SiH}$ radicals in FTIR analysis, see Figure $1 b$.

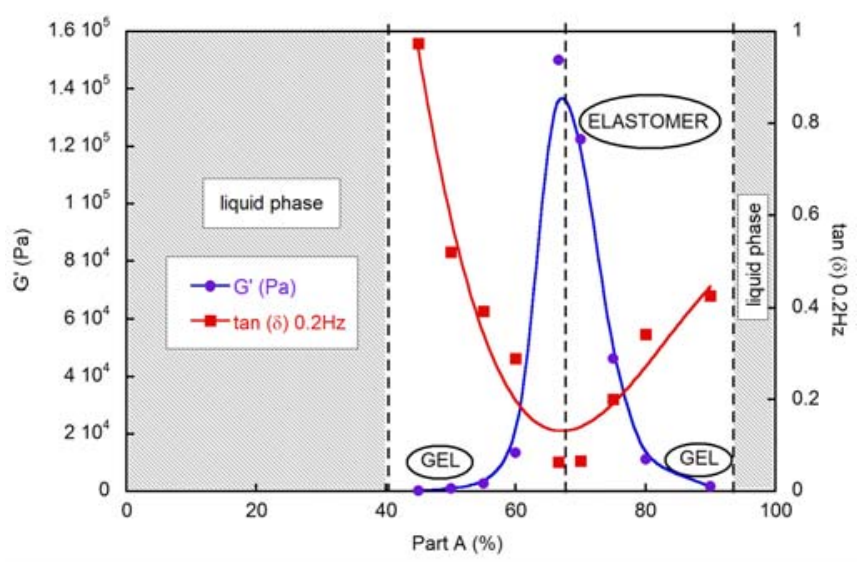

Figure 3. Stabilized end-values of G' (blue circles) and tan ( $\delta$ ) (red squares) after completion of the cure process at $65^{\circ} \mathrm{C}$, as a function of concentration of A (see Table 1), for each sample of Figure 2.
After the completion of the cure process, frequency scans between $100 \mathrm{~Hz}$ and $0.01 \mathrm{~Hz}$ were performed on each sample at different fixed temperatures. A wide damping peak was observed with a maximum between 5 and $10 \mathrm{~Hz}$, and the upper inset of Figure 4 shows the peak in $\tan (\delta)$ and its displacement with temperature (over the range $0^{\circ} \mathrm{C}$ to $80^{\circ} \mathrm{C}$ ) for the sample $50 \% \mathrm{~A}$. The lower inset shows the Arrhenius plot of this shifting in frequency of the $\tan (\delta)$ peak for the same sample $50 \% \mathrm{~A}$, which gives an activation energy Eact $=$ $10 \mathrm{~kJ} / \mathrm{mol}$ (i.e. $0.1 \mathrm{eV}$ per molecule), that we relate to the mobility of the liquid fraction inside the polymer matrix. The plot of this activation energy as a function of sample composition is given in the main part of Figure 4 and shows a similar shape to the composition plot of G' (Figure 3), with a maximum of around $250 \mathrm{~kJ} / \mathrm{mol}$ at $66-70 \% \mathrm{~A}$. That is, the harder the sample and the more cross-linked, the higher the activation energy. This is also consistent with the idea that at these percentages of $\mathrm{A}$ there is the lowest uncured liquid fraction (from components $\mathrm{A}$ and $\mathrm{B}$ ) inside the elastomeric matrix and the maximum efficiency of curing has been achieved. On the other hand, in the gel regions (i.e. around $50 \% \mathrm{~A}$ and around $90 \% \mathrm{~A}$ ), the liquid fraction is co-involved in determining the mechanical properties, with a high mobility (a low activation energy) inside a poorly reticulated matrix. To the left of the highest cross-linking efficiency (i.e. 66-70\%A) we can say that there is a decrement of $\mathrm{A}$, and to the right there is an excess of it. Similar mechanical parameters can be observed for the samples of $55 \% \mathrm{~A}$ and $90 \% \mathrm{~A}$, and also $60 \% \mathrm{~A}$ and $80 \% \mathrm{~A}$. Although the remaining liquid phase comprised of the non-reacted curing active agents should be slightly different in each gel region, this seemingly only affects the curing kinetics (slope of $G^{\prime}$ in Figure 2); causing them to be faster when component $\mathrm{A}$ is in excess (i.e. $80 \% \mathrm{~A}, 90 \% \mathrm{~A}$ ).

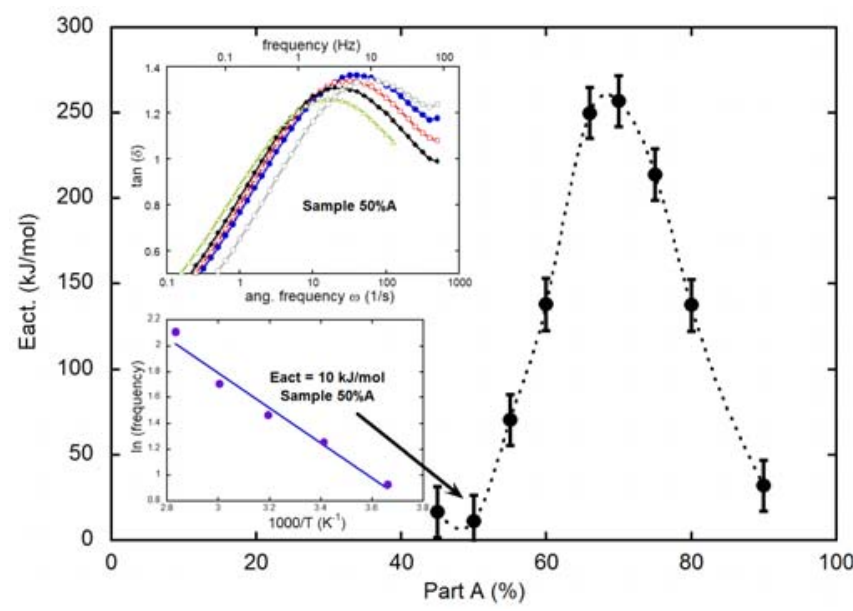

Figure 4. Change in the activation energy (Eact) of tan $(\delta)$ peak for samples between $45 \% \mathrm{~A}$ and $90 \% \mathrm{~A}$ after curing at $65^{\circ} \mathrm{C}$. Upper and lower insets: example of $\tan (\delta)$ peak and activation energy calculation for the sample $50 \% \mathrm{~A}$. Labels in the upper inset: $80^{\circ} \mathrm{C}$ (grey empty circles), $65^{\circ} \mathrm{C}$ (blue circles), $40^{\circ} \mathrm{C}$ (red empty squares), $20^{\circ} \mathrm{C}$ (black diamonds), $0^{\circ} \mathrm{C}$ (green empty triangles). 


\subsection{ELECTRICAL TREEING}

Electrical treeing tests were carried out at room temperature (RT) of $20^{\circ} \mathrm{C}$ by applying a high $\mathrm{AC}(50 \mathrm{~Hz})$ voltage to the pin electrode with the plane electrode earthed. For solid and gel samples the trees were initiated at $9 \mathrm{kV}$ with a tree being regarded as initiated by the time of the first frame that showed the existence of a tree of whatever length. These trees were then propagated at $8.0 \mathrm{kV}$. For liquid or quasi-liquid samples, the voltage was raised slowly and steadily up to this value $(8 \mathrm{kV})$ to avoid a sudden breakdown.

In the liquid region only streamers are grown, and in the elastomer region only branched tree structures are found. Gels near the gel-liquid boundary grow streamers as do the liquids, whereas gels near the gel-elastomer boundary grow both streamers and branched tree structures.

Figure 5 shows snapshots illustrating the main differences between electrical trees grown in each microstructure region (see Figure 3). The first sequence, corresponding to a quasiliquid sample (with $45 \% \mathrm{~A}$ ) on the gel-liquid boundary, shows a fast streamer projection with more than $1 \mathrm{~mm}$ length formed within 3 minutes that starts to disappear in the next frame $1 / 20$ s later. This means that the speed of propagation is faster than $20-25 \mathrm{~mm} / \mathrm{s}$. A complete and fast self-healing behaviour is observed in the order of microseconds to tenths of a second. This behaviour, which occurs with the voltage applied, we have named as a self-healing stage \#1 mechanism. In other similar samples breakdown occurred when these streamers reached the earthed bottom electrode before dissipation. No tree structures could be grown in these kinds of samples.

The second sequence shows an electrical tree growing in a gel sample $(50 \% \mathrm{~A})$. Here the growth mechanism involves the formation of near spherical cavities on the tips of filaments. To avoid confusion the reader should note that hereinafter we term the narrow tubular structures as filaments and reserve the term cavity for the wide spherical or elliptical formations to be seen at or near the filament tips. These cavities in gels are in fact slower streamers with short and wider dimensions when compared with those found in liquids $[5,7,8]$. They expand collapse and disappear as do such cavities in liquid breakdown structures $[7,9,10]$. Here they collapse and disappear in the order of a tenth of a second to a few seconds, due to a selfhealing stage \#1 mechanism. However the repetition of discharges or the longer the time a cavity receives them makes them stable. This new quasi-permanent filament adds to the fractal tree structure that is growing from the tip of the needle as the backbone of the degradation structure. The continuous shape changing observed in the whole tree therefore only occurs in the newest parts or filament tips, where the stage \#1 of self-healing is acting. After some hours or days from the end of the test (after removal of the applied voltage) these trees might suffer a second stage of self-healing we call stage \#2, which is more usually mentioned in the literature. This corresponds to the "disappearance" of part of the tree structure with a change in the refractive index in these zones [2].

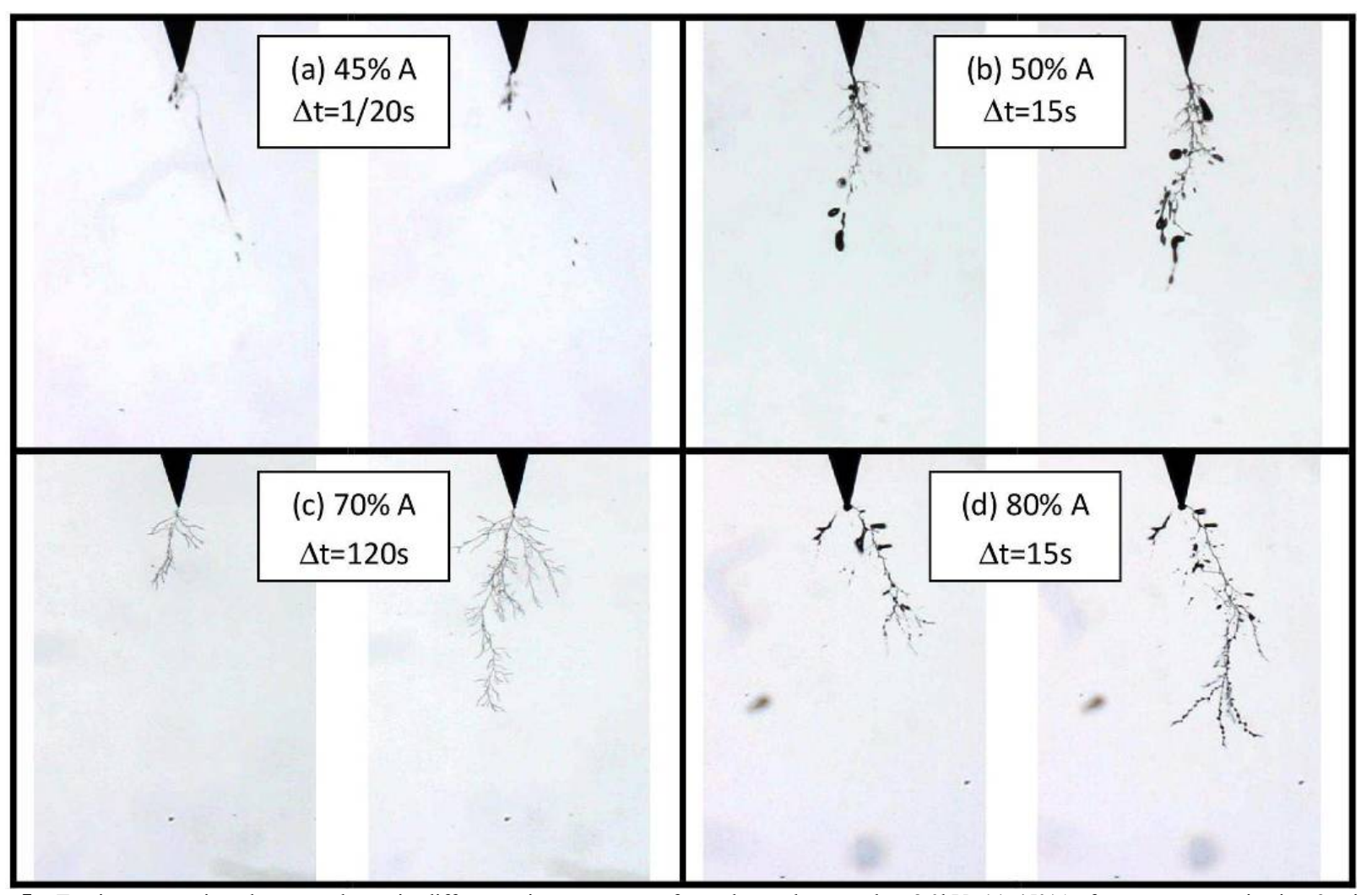

Figure 5. Treeing comparison between the main different microstructures of cured samples tested at $8.0 \mathrm{kV}$. (a) $45 \% \mathrm{~A}$ : fast streamer projection 3 min after inception and collapse in the next $1 / 20$ s - sample just gelled. (b) 50\%A: gel sample with cavity growing mechanism and self-healing stage \#1 in filament tips, with the stabilized electrical tree growing as a backbone. First image 4 min after inception. (c) $70 \%$ A: typical branched tree 30 s after inception, second image 120 s later. (d) $80 \%$ A: sample on the edge of the high A\% gel region. First image 1 min after inception, second image $15 \mathrm{~s}$ later. 
The third sequence (c) shows the behaviour of a sample cured with $70 \% \mathrm{~A}$ i.e. the elastomer composition, see Figure 3. Here a typical branch electrical tree can be observed with very thin filaments and no cavity formation. Each new portion of the tree is gained through an irreversible chemical change (i.e. chain scissions and/or further carbonization). Therefore, it is not possible for a stage \#1 self-healing mechanism to exist, although the stage \#2 mechanism might still be observed as shown later.

Finally, in the fourth sequence (d) corresponding to the second gel region of Figure 3, a cavity growing mechanism acting at the filament tips can be observed once again. This sample with $80 \% \mathrm{~A}$ has mechanical properties comparable with the samples of $60 \% \mathrm{~A}$. These samples have the largest value of $G^{\prime}$ in each of the gel regions, see Figure 3. Because of this the cavities are smaller (due to a harder network) than in the $55 \% \mathrm{~A}$ or its $90 \% \mathrm{~A}$ equivalent. However, it is still possible to distinguish both self-healing stages as with the other gel compositions.

Figure 6 shows the normalized area decay during the prompt self-healing stage \#1 of cavities that are formed in the gel samples. In order to make a valid comparison of materials of different composition we selected cavities of a similar shape and initial area for each sample condition in making this plot. It can be observed that the speed of decay follows the amount of liquid phase remaining in the sample. That is, the more the liquid phase in the sample the faster the collapse; the more the cross-linked phase and the less liquid phase inside the reticulated matrix, the slower the collapse.

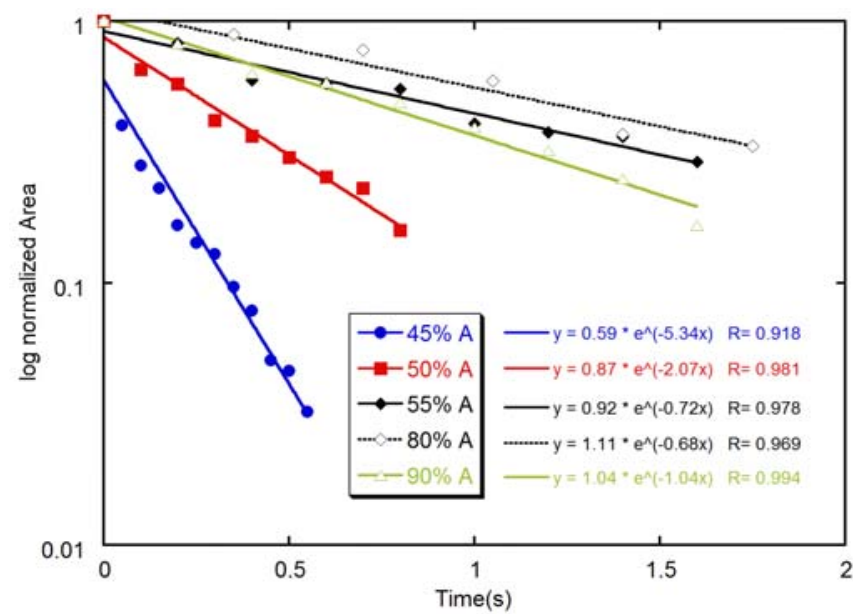

Figure 6. Decay speed of cavities during self-healing stage \#1, given as the slope of normalized area (log axis) vs. time for samples $45 \% \mathrm{~A}$ (circles), $50 \% \mathrm{~A}$ (squares), $55 \% \mathrm{~A}$ (diamonds), $80 \% \mathrm{~A}$ (empty diamonds) and $90 \% \mathrm{~A}$ (empty triangles).

Figure 7 shows the self-healing stage \#2 mechanism. The upper sequence shows the behaviour of a tree in the gel sample with $50 \% \mathrm{~A}$, with a) being the structure some seconds after removing the growth voltage of $8 \mathrm{kV}$ (i.e. voltage becomes zero), b) being the structure observed 24 hours later, and c) being the structure observed after a period of around 5 minutes of further growth at $8 \mathrm{kV}$, but again observed with the voltage set to $0 \mathrm{kV}$. The lower sequence shows the same set of observations for trees in the rubbery (elastomer) sample $(66 \% \mathrm{~A})$.

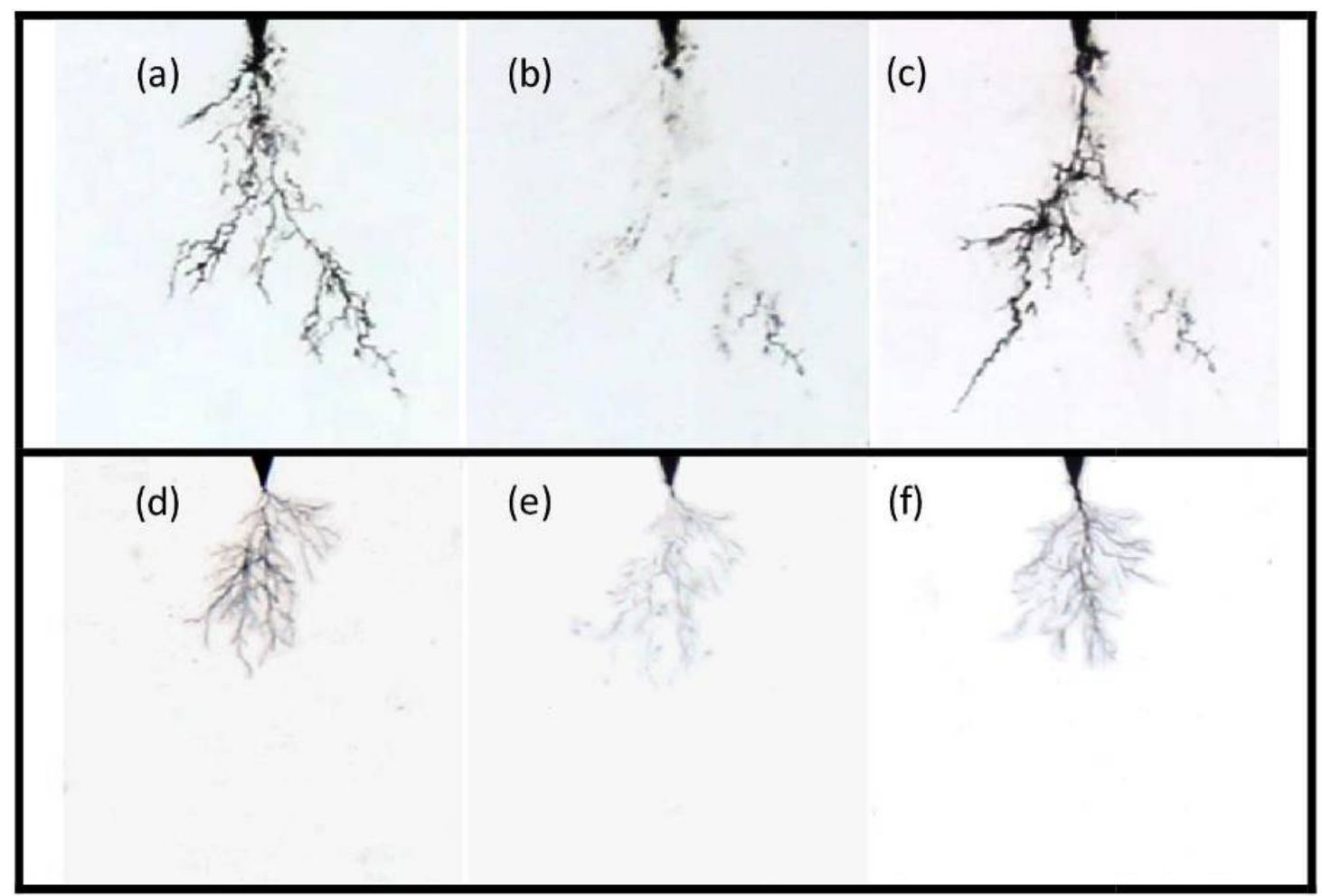

Figure 7. Self-healing stage $\# 2$ for a gel sample with $50 \% \mathrm{~A}(\mathrm{a}, \mathrm{b}, \mathrm{c})$ and a rubbery sample with $66 \% \mathrm{~A}(\mathrm{~d}, \mathrm{e}, \mathrm{f})$. a) and d): Initial tree at $0 \mathrm{kV}$ following growth at $8 \mathrm{kV} ; \mathrm{b}$ ) and e): tree after $24 \mathrm{~h}$ at $0 \mathrm{kV} ; \mathrm{c}$ ) and f): new tree at $0 \mathrm{kV}$ after further growth at $8 \mathrm{kV}$. 
In Figure 8, bar-plots summarise the average speeds of: (a) the self-healing stage \#1 mechanism, from the collapsing area of cavities against time; (b) tree growth, as the length vs. time of the underlying filaments that are not being self-healed by stage $\# 1$; and (c) streamers, as the length of the elongated cavity that develops in any direction in a fraction of time.
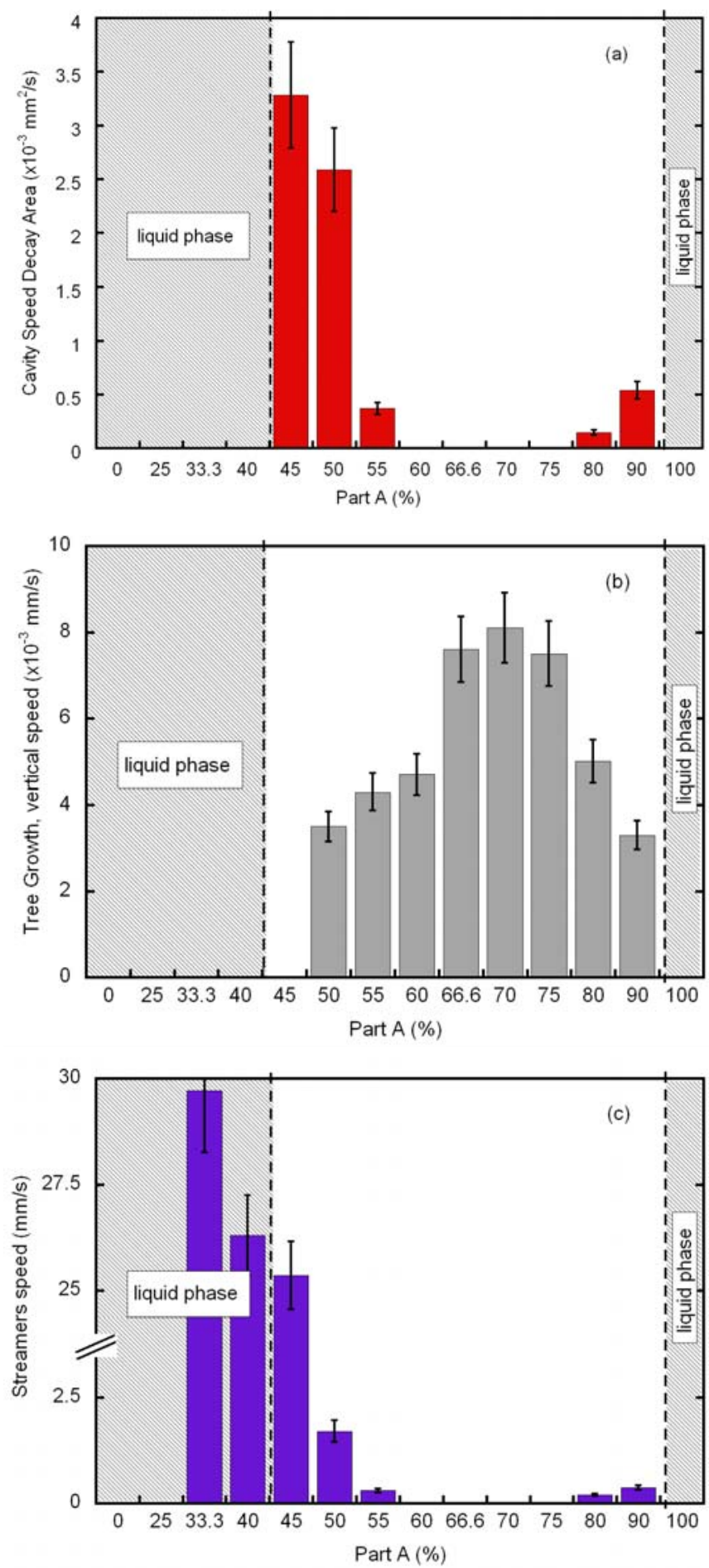

Figure 8. Bar diagrams of average speeds of: (a) cavity decay, (b) tree growth, and (c) streamer propagation, as a function of the fraction of component A.

\section{DISCUSSION}

\subsection{MICROSTRUCTURE AND CURING}

Addition is the preferred cure system employed in silicone gels and elastomers when a dielectric application is required where no by-products (such as in condensation type curing) are evolved and no shrinkage wanted $[1,11]$. In the cured silicone polymer the $\mathrm{Si}-\mathrm{O}-\mathrm{Si}$ bond angle can vary between $105^{\circ}$ and $180^{\circ}$, and the rotation is essentially free $[11,12]$ around these bonds. As a result, the chains are very flexible and occupy a rather large volume, resulting in a high free volume in the material. Silicones therefore exhibit a very low glass transition temperature $\left(\mathrm{Tg} \approx-125^{\circ} \mathrm{C}\right)$ [1]. Furthermore, low intermolecular interaction strengths result in a low melting temperature $\left(\mathrm{Tm} \approx-50^{\circ} \mathrm{C}\right)$ for the very small size crystalline fraction that might exist in these kinds of materials.

As is demonstrated in Figures 2 and 3 mixing ratios different to the $1: 1$ of the supplier's specifications generates a very rich number of different microstructures. Thus starting the pure liquid of component $\mathrm{B}(0: 1$ ratio) the viscosity of the final product increases with increasing concentration of component A up to around $45 \% \mathrm{~A}$, when the gel point is reached (at $0.2 \mathrm{~Hz}$ in a dynamic test). Above this concentration of A the real part of the shear modulus G' exceeds the imaginary part $\mathrm{G}^{\prime}$, giving a $\tan (\delta)<1$, and therefore the dimensional stability of an elastic solid. From $45 \% \mathrm{~A}$ to $60 \% \mathrm{~A}$, a gel region (Figure 3) can be defined, even though G' can vary by two orders of magnitude in this range. At higher percentages of $\mathrm{A}$, a further hardening effect is found, i.e. the curing reaction between active groups inside each component (i.e. $\equiv \mathrm{SiH}$ groups in A and vinyl end-blocked groups in B) is optimized, thereby increasing the number of cross-linking points and consequently an increase of the elastic behaviour ( $G$ ' rises). The maximum occurs between $66 \% \mathrm{~A}-70 \% \mathrm{~A}$, with $\mathrm{G}^{\prime}=1.5 \times 10^{5} \mathrm{~Pa}$ giving the physical consistency of a typical silicone rubber. This is in complete agreement with the FTIR results (Figure 1b), which indicates that $68.9 \% \mathrm{~A}$ is the curing ratio that gives the most complete consumption in cross-links. An excess of part A ( $=\mathrm{SiH}$ groups) beyond this optimal ratio results in an increasing amount of $A$ that cannot be reacted due to the lower amount of part B (vinyl groups) available, and hence a decreasing amount of cross-linking points. The non-reacted liquid remains trapped inside the poorly reticulated matrix, which is swelled, sticky, and soft, resulting in a second gel region (from $80 \% \mathrm{~A}$ to $90 \% \mathrm{~A}$ ) similar to that at the lower concentrations (45\%A to $60 \% \mathrm{~A}$ ). Finally, above 90 $95 \% \mathrm{~A}$, there is a very low concentration of vinyl end-blocked groups (carried on $\mathrm{B}$ ) that can react with an excess of $\equiv \mathrm{SiH}$ oligomers (on A). The low density of these reactions is not enough to gel the sample and therefore it remains as a viscous liquid, and behaves as such.

At the gel and elastomer compositions the liquid fraction inside the reticulated matrix acts as a plasticizer, even at its lowest amount for the most cross-linked samples between 66$70 \%$ A. These low molecular weight chains (LMW), can move throughout the network, and their diffusion or mobility is thermally activated, with the ease of motion depending on the 
cross-linking density of the solid phase. In addition, they are also partly responsible for the low $\mathrm{Tg}$ temperature in these materials. The activation energy (Eact) determined from the shifting of the high temperature tan $(\delta)$ peak during frequency scans (Figure 4) can be attributed to this molecular mobility. For a sample in the gel region Eact lies between 10 and 20 $\mathrm{kJ} / \mathrm{mol}$ ( 0.1-0.2 eV per relaxing molecule), while for the rubber or elastomeric region it reaches $250 \mathrm{~kJ} / \mathrm{mol}$. This order of magnitude increase reflects the big increase in hindrance to the mobility of the liquid fraction produced by the high crosslinking density.

A comparison of the two gel regions (i.e. $55 \% \mathrm{~A}$ with $90 \% \mathrm{~A}$, and, $60 \% \mathrm{~A}$ with $80 \% \mathrm{~A}$ ), shows that their mechanical properties are similar, although the residual liquid phase (having the non-reacted curing active agents) might be slightly different in each case. The FTIR spectra shows that the only difference between pure A and pure B is the presence of the absorption peak at $2127 \mathrm{~cm}^{-1}$ related to the $\equiv \mathrm{SiH}$, which is only present in pure A (Figure 1a). This absorption band is sensitive to the curing reaction in which the $\equiv \mathrm{SiH}$ is consumed after mixing $\mathrm{A}$ and $\mathrm{B}$ together (Table 1) in agreement with the reaction mechanism. As shown in Figure $1 \mathrm{~b}$ all of the $\equiv \mathrm{SiH}$ radical is consumed in the $50 \% \mathrm{~A}$ mixture, however this is insufficient to consume all the vinyl groups on component $\mathrm{B}$ and give the maximal cross-linking, which the mechanical response shows occurs between $66 \% \mathrm{~A}$ and $70 \% \mathrm{~A}$, but FTIR confirms should be at $68.9 \% \mathrm{~A}$ where the reaction is optimized (see equation (1)). This ratio of $\mathrm{A}$ to $\mathrm{B}$ has the highest reaction efficiency with all the $\equiv \mathrm{SiH}$ radicals and vinyl groups reacting. Finally, samples with $80 \% \mathrm{~A}$ and $90 \% \mathrm{~A}$ have an excess of component $\mathrm{A}$, therefore the radical is not fully consumed at the end of the reaction. The FTIR results (Figure $1 b)$, shows that the amount of unreacted $\equiv \mathrm{SiH}$ increases for concentration above $68.9 \% \mathrm{~A}$ follows equation (1).

\subsection{ELECTRICAL TREEING TESTS}

The phenomenon of electrical treeing in solid dielectrics has been intensely studied for materials with a range of chemical composition and physical morphologies (e.g. [1326]), including silicone rubber [27]. The equivalent breakdown phenomena in liquids i.e. branched streamers, has also received a lot of attention (e.g. [5, 7-10, 28-30]). However the behaviour of gels has received little attention [24]. The data obtained here indicates that the Low Molecular Weight (LMW) liquid content of the samples is instrumental in determining the structure and behaviour of the electrical trees in the gel samples whereas in the silicone rubber elastomer the flexibility of the 3D matrix becomes important.

In the $45 \% \mathrm{~A}$ sample of Figure $5 \mathrm{a}$ the tree structure is dominated by an unbranched leading streamer that grows rapidly ( $\sim 25 \mathrm{~mm} / \mathrm{s}$ : see Figure $8 \mathrm{c})$. This structure has almost disappeared within $1 / 20 \mathrm{~s}$. There is however a small branched structure near to the needle electrode that is retained. In the $50 \% \mathrm{~A}$ sample the branched structure extends much further into the sample and ellipsoidal bubble cavities are more evident at and near the tips of the branches. The filamentary structures are similar to those grown from positive needles in liquids [8, 28], where they have been attributed to the avalanche extraction of electrons from the liquid with the structure extending progressively into the sample [28]. They have also been observed from positive points in a gel [2]. However the appearance of bubble cavities along the filamentary branches, at their tips and even isolated from the branches, clearly evident at 50\%A (Figure 5b), are more typical of negative point structures in liquids $[5,7,8]$. In [2] it has been suggested that in $\mathrm{AC}$ fields the tree structure starts on the negative half-cycle with the formation of bubble cavity. This is retained into the following cycles and rapidly extends into the material in the form of a streamer. It is presumed that initial electron injection heats the liquid component allowing vaporisation and the formation of cavities capable of supporting gas discharges within themselves. In our case the needle voltage and pin radius $(8 \mathrm{kV}$ and $5 \mu \mathrm{m})$ will give a much lower needle field than those used in [2] $(12.5 \mathrm{kV}$ and $1.5 \mu \mathrm{m})$ and our AC structures grow more slowly on average $(\sim 3 \mathrm{~mm} / \mathrm{s}$ at the manufacturers gel composition of $50 \% \mathrm{~A}$ compared to $\sim 300 \mathrm{~mm} / \mathrm{s}$ in [2]). In this case bubble cavity formation may be expected as a result of equilibration between the internal gas and electrostatic pressure and the external LMW liquid hydrostatic pressure $[9,10]$. The resulting structures in these conditions seem to be a combination of positive point filaments and negative point cavities such as found in some liquids [7, 9], although some streamers are observed with a lower growth rate of $\sim 1 \mathrm{~mm} / \mathrm{s}$, (see Figure $8 \mathrm{c}$ ) compared both to the samples with a higher LMW liquid fraction and to the branched filaments at the same composition. The increasing density of matrix crosslinks and decrease of the LMW liquid fraction is reflected in an increased growth rate of the filamentary structure (see Figure 8b), filament length, retention time (compare Figure 5a and 5b), and a slower decay rate for the bubble cavities that form as part of the structure (see Figure 6).

The second gel region, between $80-90 \% \mathrm{~A}$, has similar combined bubble cavity/filamentary structures as those in the first gel region (50-60\%A), see Fig.5. Their cavity speed decay is nearly the same as that at 55\%A (see Fig.6). Both gel regions possess a partly cross-linked matrix and these results indicate that the amount of cross-linking in the matrix plays a role in the collapse rate of the cavities.

Most of the cavities that isolate and collapse are produced at the tips of the tree, although this is not always the case. Figure 6 shows that the rate at which the projected bubble area reduces is proportional to the cross-sectional bubble area, $A_{c}$,

$-\frac{d A_{c}}{d t}=k \times A_{c}$

Integration of equation (2) gives:

$A_{c}=c \times e^{-k t}$

Here $k$ and $c$ are constants and $t$ is time. The fit of the data to equation (3) is shown by the straight lines in Figure 6. The 
growth and collapse of cavities formed during high voltage experiments in liquids have been described in terms of the kinetics of a bubble under the action of a difference between the internal and external pressure [5, 29]. In these models the rate of collapse speeds up as the radius reduces, and it is predicted that the rate of reduction in radius is proportional to $\mathrm{r}^{-3 / 2}$ for spheres [29] and $\mathrm{r}^{-1}$ for right circular cylinders [5]. In turn these predictions imply that a power of $A_{c}$ should show a linear relationship with time rather than the $\log$ of $A_{c}$ as in Figure 6. Thus it appears that the collapse of the bubble cavities in gels is not controlled by a pressure differential as in liquids and a more complex mechanism is involved. One possibility is that the bubble cavity collapse is due to the reduction in its internal temperature once discharging within it ceases. The volume would reduce and the internal gas recondense into its liquid state. Since the rate of loss of heat would be proportional to the surface area $A_{s}$ and hence to $A_{c}$ (as both are proportional to $\mathrm{r}^{2}$ ) then equation (2) would result. Any surplus gas would diffuse through the surface following a similar equation. If this is the case then either the fully crosslinked matrix inhibits the heat loss from the cavities or its high shear modulus (see Figure 2) slows down the structural rearrangement required on collapse.

Branched filamentary electrical tree structures are observed in the elastomeric region (see Figure 5c and Figure 7 (d-f) that are similar in form to those found in other solid insulating dielectrics $[13,14]$. Bubble cavities are not formed because of the low LMW liquid fraction in this region. This is consistent with other work [27]. The rate of growth of the branched structures goes through a peak at the composition of maximum cross-linking and least LMW liquid fraction, $68.9 \% \mathrm{~A}$ (see Figure $8 \mathrm{~b}$ ). These features indicate that their propagation mechanism is similar to that of other solids; i.e. it is driven by discharges in the tubes of the filamentary structure [27]. The near symmetrical slowing down of the growth rate as the composition moves away from its peak at the optimal cross-linking density suggest that it is due to the ingress of the small amount of LMW liquid fraction into the tubes, which can temporarily prevent the discharging as is known from the inhibition of electrical trees in polyethylene by oil impregnation [13]. The filamentary structure in this region is a permanent feature that is retained following the removal of the applied voltage (see Figure 7 (d-f)). Unlike more rigid insulators such as epoxy resins and polyethylene however, the flexible elastic nature of the matrix allows the structure to contract once the voltage is removed [4], a phenomenon that we have called stage \#2 self-healing. This would indicate that the tubes that form the filamentary structure are characterised by a re-arrangement of the crosslinks on their surface giving a framework that is in tension from electrostatic forces and internal gas pressure, during voltage application, and which relaxes slowly as the tension is released. The structure also fades, i.e. there is a change in refractive index, (see Figure 7e). Since this is also observed in gel samples, Figure $7 \mathrm{~b}$, it is likely that it is due to ingress of the LMW liquid fraction into the filamentary structure.

Stage 2 \# self-healing has also been observed for the filamentary structures grown in gels near to the elastomer-gel boundary indicating the existence of a similar framework in their structure. Here however there is a loss of memory such that the structure is not completely the same when the voltage is re-applied (see Figure 7 (a-c) i.e. many branches of the original structure have been lost, suggesting that some parts of the filamentary structure can fragment and re-arrange when the matrix is not fully cross-linked. Nonetheless some filamentary structure is retained particularly near to the point electrode which has experience discharging for the longest time, and this can be regarded as a form of irreversible degradation such as observed in [2]. It is clear from our results that the manufacturer's recommended composition of $50 \% \mathrm{~A}$ represents a good compromise. It has a low growth rate for streamers (Figure 8c) compared to gels with a lower concentration of $\mathrm{A}$, and a low growth rate for filamentary structures compared to gels with higher percentages of A (Figure $8 \mathrm{~b}$ ). It also has a significant amount of self-healing both during voltage application and following voltage removal (Figure 7 (a-c)).

\section{CONCLUSION}

We have shown that electrical trees grown in silicone gels at lower ac voltages than those adopted by other workers combine features of tree structures from both liquids and solids. At the manufacturers recommended composition $(50 \% \mathrm{~A})$ there is a filamentary branched backbone such as occurs in solids but around its periphery are bubble-shaped cavities that may collapse and separate from the backbone just as in liquid streamers. Their presence and rate of collapse has been shown to be dependent on the fraction of liquid inside the cured gel. When there is no liquid phase as in the elastomer they do not exist. However it has been shown that the rate of collapse of the bubble cavities does not follow the behaviour expected from liquid hydrodynamics. Instead a relationship is obeyed that indicates that the controlling factor may be the loss of heat through the cavity surface and/or diffusion of internal bubble gases through it. It has also been shown that this limited self-healing that occurs during voltage application is accompanied by a more substantial self-healing following voltage removal. This behaviour has also been noted to some extent in the elastomer and has been associated with the elasticity of the filamentary part of the electrical tree and ingress of the liquid content. These self-healing properties are favourable to the use of silicone gels for high voltage applications in areas where the softness of the gel is not a drawback.

Special care must be taken if the mechanical properties and self-healing behaviour are needed to be between some narrow ranges. An error of $5-10 \%$ of component $\mathrm{A}$ in a desired gel, such as that recommended by the manufacturer can produce either an uncured system (liquid) or a harder material without self-healing (depending respectively upon whether the error is towards smaller or larger percentages). 


\section{ACKNOWLEDGMENT}

This work was supported by the Department of Engineering, University of Leicester, UK, the Argentinean Science Research Council (CONICET), the ANPCyT and the National University of La Plata (UNLP) of Argentina.

\section{REFERENCES}

[1] A. Colas, Chimie Nouvelle, from Dow Corning technical brochures Vol. 8 (30), pp. 847, 1990.

[2] T. M. Do, O. Lesaint and J.-L. Augé, "Streamers and Partial Discharge Mechanisms in Silicone Gel Under Impulse and AC Voltages", IEEE Trans. Dielectr. Electr. Insul., Vol. 15, No. 6, pp. 1526-1534, 2008.

[3] G. Finis and A. Claudi, "On the Electric Breakdown Behavior of Silicone Gel at Interfaces", IEEE Trans. Dielectr. Electr. Insul., Vol.15, No. 2, pp. 366-373, 2008.

[4] S. J. Dodd, L. M. Salvatierra, L. A. Dissado, E. E. Mola, "Electrical Trees in Silicone Gel: A Combination of Liquid and Solid Behaviour Patterns", 2013 Annual Report Conference on Electrical Insulation and Dielectric Phenomena, pp. 1018-1021 [IEEE Conference on Electrical Insulation and Dielectric Phenomena (CEIDP), Shenzhen, China, Oct. 2013].

[5] P. Gournay and O. Lesaint, "On the gaseous nature of positive filamentary streamers in hydrocarbon liquids. II: Propagation, growth and collapse of gaseous filaments in pentane", J. Phys. D: Appl. Phys., Vol. 27, pp. 2117-2127, 1994.

[6] F. O. Stark, J. R. Falenda, A. P. Wright, Silicones, in Comprehensive Organometallic Chemistry, G. Wilkinson, F. G. Sone, E. W. Ebel Eds., Vol. 2, 305, Pergamon Press, Oxford, UK, 1982.

[7] A. Beroual, M. Zhan, A. Badeut, K.Kist, A. J. Schwabe, H. Yamashita, K. Yamazawa, M. Danikas, W. G. Chadband, Y. Torshin, "Propagation and structure of streamers in liquid dielectrics", IEEE EI-Mag., Vol. 14 (2), pp. 16-17, 1998

[8] H. Akiyama, "Streamer discharges in liquids and their applications", IEEE Trans. Dielectr. Electr. Insul., Vol. 7, pp. 646-652, 2000.

[9] P. K. Watson, W. G. Chadband, M. Sadeghzadeh-Araghi, "The role of electrostatic and hydrodynamic forces in the negative point breakdown in Liquid Dielectrics", IEEE Trans. Dielectr. Electr. Insul., Vol. 26, pp. 543-559, 1991.

[10] T. Aka-Ngnui, A. Beroual, "Bubble dynamics and transition into streamers in liquid dielectrics under a high divergent electric field", J. Phys. D: Appl. Phys., Vol. 34, pp. 1408-1412, 2001.

[11] F. Gubbels, Chapter 2: Silicones in Industrial Applications in Inorganic Polymers, Nova Science Publishers, by Roger De Jaeger and Mario Gleria Eds. pp. 61-161, 2007.

[12] E. B. Baker, A. J. Barry, M. J. Hunter, "Dielectric constants of dimethyl siloxane polymers", Ind. \& Eng. Chem., Vol. 38; pp. 1117-1120, 1946.

[13] L. A. Dissado and J. C. Fothergill, Electrical Degradation and Breakdown in Polymers, IEEE Materials and Devices Series 9, Ed. G.C. Stevens (Ed.), P. Peregrinus Ltd. London, UK, 1992.

[14] L. A. Dissado, "Understanding Electrical Trees in Solids: From Experiment to theory", IEEE Trans. Dielectr. Electr. Insul., Vol. 9, pp. 483-497, 2002.

[15] C. Laurent, G. Teyssedre, S. Le Roy, F. Baudoin, "Charge Dynamics and its energetic features in Polymeric materials", IEEE Trans. Dielectr. Electr. Insul., Vol. 20-2, pp. 357-381, 2013.

[16] G. C. Montanari, "Bringing an Insulation to failure: The role of space charge", IEEE Trans. Dielectr. Electr. Insul., Vol. 18, pp. 339-364, 2011.

[17] A. S. Vaughan, I. L. Hosier, S. J. Dodd and S. J. Sutton, "On the structure and chemistry of electrical trees in polyethylene", J. Phys. D: Appl. Phys., Vol. 39, No.5, pp. 962-978, 2006.

[18] Xiangrong Chen, Yang Xu, Xiaolong Cao, S. J. Dodd, L. A. Dissado, "Effect of Tree Channel Conductivity on Electrical Tree Shape and Breakdown in XLPE Cable Insulation Samples", IEEE Trans. Dielectr. Electr. Insul., Vol. 18, Issue 3, pp. 847-860, 2011.

[19] K. Wu and L. A. Dissado, "Model for Electrical Tree Initiation in Epoxy Resin”, IEEE Trans. Dielectr. Electr. Insul., Vol. 12, pp. 655-668, 2005.
[20] J. Wu, T. Iizuko, K. Monden, T. Tanaka, "Characteristics of initial trees of 30 to $60 \mu \mathrm{m}$ length in Epoxy/Silica nanocomposites", IEEE Trans. Dielectr. Electr. Insul., Vol. 19, pp. 312-320, 2012.

[21] L. A. Dissado, J. C. Fothergill, N. Wise, A. Willby and J. Cooper, "A deterministic model for branched tree structures in the electrical breakdown of solid polymeric dielectrics", J. Phys. D: Appl. Phys., 33, pp. L109-L112, 2000.

[22] T. Tanaka, "Buds for treeing in epoxy nanocomposites and their possible interaction with nano-fillers", Proc. 10th IEEE-ICSD (Potsdam), paper no. B1-1, pp. 159-162, 2010.

[23] N. M .Chalashkanov, S. J. Dodd, J. C. Fothergill, L. A. Dissado, "On the Relationship between Bulk Charge Transport and Electrical Breakdown Mechanisms in Epoxy Resins", Proc. 22nd NORDIC Insulation Symposium -Nord-IS (ISBN 978-952-15-2562-9), June 13-15, Tampere, Finland, pp. 29-32, 2011

[24] S. J. Dodd, L. A. Dissado, J. V. Champion and J. M. Alison, "Evidence for deterministic chaos as the origin of electrical tree structures in polymeric insulation", Phys. Rev. B., 52, pp. 16985-16988, 1995.

[25] L. A. Dissado, "Deterministic Chaos in Electrical Breakdown: Does it occur and what can it tell us", IEEE Trans. Dielectr. Electr. Insul., Vol. 9, pp. 752-762, 2002.

[26] J. V. Champion and S. J. Dodd, "Systematic and reproducible partial discharge patterns during electrical tree growth in an epoxy resin", J. Phys. D: Appl. Phys., Vol. 29, pp. 862-868, 1996.

[27] B. X. Du, Z. L. Ma, Y. Gao, T. Han, "Effect of ambient temperature on electrical treeing characteristics in silicone rubber", IEEE Trans. Dielectr. Electr. Insul., Vol. 18, pp. 401-407, 2011.

[28] R. E. Hebner, E. F. Kelley, E. O. Forster, G. J. Fitzpatrick, "Observation of pre-breakdown phenomena in liquid hydrocarbons: II Non-uniform field conditions", IEEE Trans. Dielectr. Electr. Insul., Vol. 20, pp. 281292, 1985

[29] P. K. Watson, W. G. Chadband, W. Y. Mak, "Bubble growth following a localised electrical discharge and its relationship to the breakdown of triggered spark gaps in liquids", IEEE Trans. Dielectr. Electr. Insul., Vol. 20, pp. 275-280, 1985.

[30] N. Hozumi, T. Okamoto, H. Fukugawa, "Simultaneous measurement of microscopic image and discharge pulses at the moment of electrical tree initiation”, Jap. Jn. of Appl. Phys., Vol. 27, pp. 572-576, 1988.

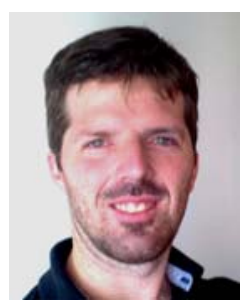

Lucas M. Salvatierra was born in Rosario, Argentina in 1979. He received the B.Sc. degree from the Pontific Catholic University of Argentina in 2001, and his Ph.D. degree from the National University of Rosario, Argentina in 2005. He is currently a research member staff of the Argentinean Science Research Council (CONICET) and he is Lecturer at the Faculty of Chemistry and Eng. of the Pontific Catholic University of Argentina. His main research interest lies with both Dielectric Materials and Ageing Phenomena, and Granular Dynamics subjected to high electric fields.

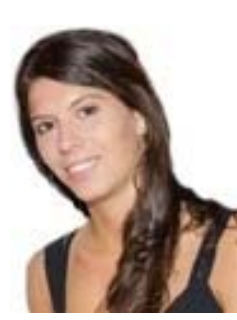

Laura I. Kovalevski was born in 1986 in Rosario, Argentina. She received the B.Sc. degree from the Pontific Catholic University of Argentina in 2009 and is presently a Ph.D. fellow of the Argentinean Science Research Council (CONICET) at the National University of La Plata, Argentina. She is currently engaged in research on ageing process and the dielectric breakdown of insulating materials both solids and liquids and her interest also includes the characterization of the self-healing in silicone gels.

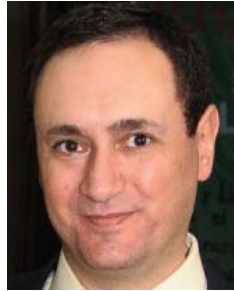

Pablo L. Dammig Quiña was born in Darregueira, Argentine in 1973. He received the Electronic Engineering degree from Universidad Nacional del Sur, Bahía Blanca, Buenos Aires, Argentina in 2001 and Ph. D. degree in Exact Sciences from Universidad Nacional de La Plata, Argentine in 2010. Since 2010 he is a research member staff of the Argentinean Science Research Council (CONICET) 


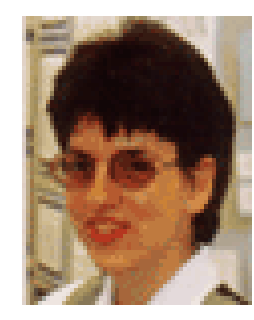

Isabel M Irurzun is a physics lecturer in the Faculty of Science, University of La Plata, Argentina. She is also an adjoint researcher of the Argentinean Science Research Council and an invited researcher at the Universities of Valencia (Spain), Hannover (Germany) and Cambridge (UK).

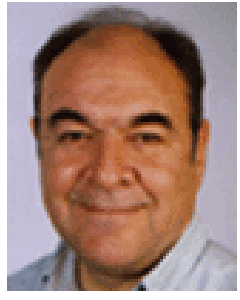

Eduardo E Mola is Head Professor of Physical Chemistry in the Faculty of Science, University of La Plata, Argentina. He is also a principal researcher of the Argentinean Science Research Council, as well as an invited professor at the Universities of Liverpoo (UK), La Florida (USA), Puerto Rico (USA), Cambridge (UK) and Leicester (UK).

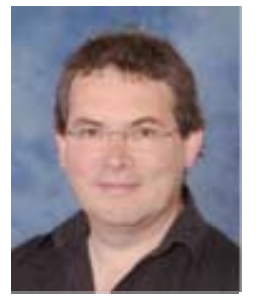

Steve J. Dodd was born in Harlow, Essex in 1960. He received the B.Sc. (Hons) Physics degree in 1987 and the Ph.D. degree in physics in 1992, both from London Guildhall University, UK and remained at the University until 2002 as a Research Fellow. He joined the University of Southampton in 2002 as a Lecturer in the Electrical Power Engineering Group in the School of Electronics and Computer Science and then the University of Leicester in the Electrical Power and Power Electronics Research Group in the Department of Engineering in 2007 as a Senior Lecturer. His research interests lie in the areas of light scattering techniques for the characterization of polymer morphology, electrical treeing breakdown process in polymeric materials and composite insulation materials, electroluminescence and its relationship with electrical and thermal ageing of polymers, characterization of liquid and solid dielectrics and condition monitoring and assessment of high voltage engineering plant. His research has attracted funding from the electrical generation and transmission industries. He has published 26 papers, 55 conference papers and contributed to two books.

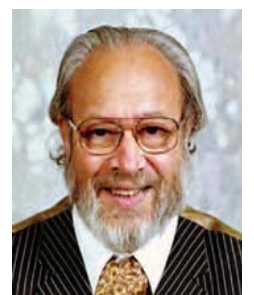

Len A. Dissado (F’06) was born in St. Helens, Lancashire, U.K. on 29 August 1942. He was educated in Thomas Linacre Technical School, Wigan, Lancashire, 1953-1960, gaining a State Scholarship for University Entry in 1959 . He graduated from University College London with a 1st Class degree in chemistry in 1963 and was awarded the Ph.D. degree in theoretical chemistry in 1966 and the D.Sc. degree in 1990. After rotating between Australia and England twice he settled in at Chelsea College in 1977 to carry out research into dielectrics. His interest in breakdown and associated topics started with a consultancy with STL that begun in 1981. Since then he has published many papers and one book, together with John Fothergill, in this area. In 1995 he moved to The University of Leicester, and was promoted to Professor in 1998. He has been a visiting Professor at The University Pierre and Marie Curie in Paris, Paul Sabatier University in Toulouse, Nagoya University, and NIST at Boulder Colorado. He has given numerous invited lectures, the most prestigious of which was the Whitehead lecture at CEIDP 2002 in Cancun, Mexico. Currently he is an Associate Editor of IEEE Transactions on Dielectrics and Electrical Insulation and Chair of the DEIS Publications Committee. He was awarded the degree of Doctuer Honoris Causa by the Universite Paul Sabatier, Toulouse, France, in 2007, and was made an Honorary Professor of XJTU, Xi'an, China in 2008. He was also made an international academic committee of SKLEIPE of XJTU in 2010. 